\title{
Structured reporting has the potential to reduce reporting times of dual-energy x-ray absorptiometry exams
}

Su Hwan Kim ${ }^{1 \dagger}$, Lara M. Sobez ${ }^{1 \dagger}$, Judith E. Spiro ${ }^{1}$, Adrian Curta ${ }^{1}$, Felix Ceelen², Eric Kampmann ${ }^{3}$, Martin Goepfert ${ }^{1}$, Raphael Bodensohn ${ }^{4}$, Felix G. Meinel ${ }^{5}$, Wieland H. Sommer ${ }^{1}$, Nora N. Sommer ${ }^{1+}$ and Franziska Galiè ${ }^{1^{*+}}$

\begin{abstract}
Background: In recent years, structured reporting has been shown to be beneficial with regard to report completeness and clinical decision-making as compared to free-text reports (FTR). However, the impact of structured reporting on reporting efficiency has not been thoroughly evaluted yet. The aim of this study was to compare reporting times and report quality of structured reports (SR) to conventional free-text reports of dualenergy x-ray absorptiometry exams (DXA).
\end{abstract}

Methods: FTRs and SRs of DXA were retrospectively generated by 2 radiology residents and 2 final-year medical students. Time was measured from the first view of the exam until the report was saved. A random sample of DXA reports was selected and sent to 2 referring physicians for further evaluation of report quality.

Results: A total of 104 DXA reports (both FTRs and SRs) were generated and 48 randomly selected reports were evaluated by referring physicians. Reporting times were shorter for SRs in both radiology residents and medical students with median reporting times of 2.7 min (residents: 2.7, medical students: 2.7) for SRs and 6.1 min (residents: 5.0, medical students: 7.5) for FTRs. Information extraction was perceived to be significantly easier from SRs vs FTRs $(P<0.001)$. SRs were rated to answer the clinical question significantly better than FTRs $(P<0.007)$. Overall report quality was rated significantly higher for SRs compared to FTRs $(P<0.001)$ with $96 \%$ of SRs vs $79 \%$ of FTRs receiving high or very high-quality ratings. All readers except for one resident preferred structured reporting over free-text reporting and both referring clinicians preferred SRs over FTRs for DXA.

Conclusions: Template-based structured reporting of DXA might lead to shorter reporting times and increased report quality.

Keywords: X-rays, Clinical decision-making, Decision trees, Quality improvement, Bone density

\footnotetext{
* Correspondence: franziska.schoeppe@gmail.com

${ }^{\dagger}$ Su Hwan Kim and Lara M. Sobez shared first authors (contributed equally).

${ }^{\dagger}$ Nora N. Sommer and Franziska Galiè shared last authors (contributed equally).

'Department of Radiology, University Hospital, LMU Munich, Munich,

Germany

Full list of author information is available at the end of the article
}

C C The Author(s). 2020 Open Access This article is licensed under a Creative Commons Attribution 4.0 International License, which permits use, sharing, adaptation, distribution and reproduction in any medium or format, as long as you give appropriate credit to the original author(s) and the source, provide a link to the Creative Commons licence, and indicate if changes were made. The images or other third party material in this article are included in the article's Creative Commons licence, unless indicated otherwise in a credit line to the material. If material is not included in the article's Creative Commons licence and your intended use is not permitted by statutory regulation or exceeds the permitted use, you will need to obtain permission directly from the copyright holder. To view a copy of this licence, visit http://creativecommons.org/licenses/by/4.0/. The Creative Commons Public Domain Dedication waiver (http://creativecommons.org/publicdomain/zero/1.0/) applies to the data made available in this article, unless otherwise stated in a credit line to the data. 


\section{Introduction}

In previous years, there have been efforts by several international radiological societies to improve the quality of radiological reports through structured reporting [15]. Several studies have shown that structured reports (SR) tend to be more complete and may contribute to better clinical decision-making compared to conventional free-text reports (FTR) [6-13]. Structured reporting may be especially useful in highly standardized exams, e.g. cranial MRI scans in multiple sclerosis patients [14] or videofluoroscopic exams [15]. Structured reporting has also been shown to be beneficial in examinations with complex criteria which need to be provided for referring physicians (e.g. in oncologic imaging for staging of rectal cancer [2], hepatocellular carcinoma [13], pancreatic carcinoma [3] or diffuse large B-cell lymphoma [16]). However, there is also an ongoing debate on potential disadvantages of structured reporting, such as the risk of oversimplification [17], distraction by additional software [18], and the challenge of integrating structured reporting tools into the clinical workflow [19].

Another aspect of the debate is the matter of time with suspected prolonged reporting times for SRs, especially in the transition phase from current free-text reporting practices to structured reporting $[1,20]$. One concern is that productivity might be reduced because of more time spent looking at the template rather than the study [21]. To date, there are only a few studies that evaluated the impact of structured reporting on reporting times, one for leg-length discrepancy measurements [22], one in the area of emergency radiology [23] and another one on mammography and ultrasound in breast cancer patients [24] - all of them showing the potential of improved reporting times when using structured reporting. Nevertheless, as a change in workflow from the current practice of creating FTRs using speech recognition systems to creating SRs is likely to take some time, a good study design is necessary to evaluate the potentially time-saving effect of structured reporting. It may therefore be advisable to focus on a very standardized exam with limited complexity such as the dual x-ray absorptiometry exam (DXA). Although there is a continuous discussion on whether alternative types of bone densitometry measurements, such as quantitative computer tomography (QCT), might be superior to DXA $[25,26]$, DXA is still viewed as the international gold standard for bone mineral density measurements by many authors [27-30].

The International Society for Clinical Densitometry (ISCD) has published a best practice guideline for DXA reporting [31] and provided an adult DXA sample report that follows these official positions. A recent study revealed that major errors in DXA reporting are very common and their occurrence can be reduced drastically when a reporting template in accordance with these ISCD suggestions is implemented [32]. In addition, the latest 2019 ISCD Official Positions specify detailed requirements of baseline and follow-up DXA reports each [33].

However, it has yet to be shown if structured reporting of DXA exams does not only improve report accuracy but is also time-saving.

Therefore, we aimed at comparing reporting times of SRs compared to FTRs for DXA while at the same time evaluating completeness of information, facilitation of information extraction and overall quality.

\section{Materials and methods}

Patient selection and study design

Retrospectively, 26 DXA scans with acquisition dates between April 1st and June 30th, 2016 were randomly selected from the 100 most recent scans in our institutional radiology information system. Images of the femur and lumbar spine were acquired during the same session, using Lunar Prodigy (GE Healthcare, Chicago, USA).

For most patients, the referring physicians requested the DXA for clinical surveillance of osteoporosis and in some cases to confirm osteoporosis. Exams were included in the study if both the femur and the lumbar vertebral column had been measured on the same day and at least one previous scan acquired at our hospital was available for comparison. Our patient sample consisted of 3 men (mean age: 76 years, min. 67, max. 90) and 23 women (mean age: 70 years, min. 35, max. 86). The study was approved by the institutional review board. Informed consent was waived by the review board as data were analyzed anonymously as part of our department`s internal quality management program.

\section{Sample size calculations}

Sample size calculations were performed based on expected differences of reporting times for DXA exams of conventional FTRs compared to SRs. Since there have been no prior studies on this issue, mean reporting times of FTRs for DXA by the residents that had been generated during the training phase were applied as a rough estimate. Based on the average reporting time of $318 \mathrm{~s} \pm 75$ $\mathrm{SD}$, a reduction of $20 \%$ in reporting time for a SR was considered as a relevant effect size. Assuming those differences between both reporting types, a sample size of $N=$ 44 (22 per group) would be required to achieve a power of $80 \%$ at a level of significance $\alpha=0.05$. To account for the possibility of overestimating the reduction in reporting time for SRs, an extra of 8 reports were added, leading to a final sample size of $N=52$ (26 per report type). This 
sample size was used for both residents and medical students, adding up to 104 reports in total.

\section{Template for structured reporting}

The reporting template we implemented was designed using software from Smart Reporting GmbH (Munich, Germany). This online software allows users to design templates for creating SRs. It consists of point-and-click menus representing a pre-defined decision tree. The template was created based on international guidelines by the ISCD and the National Osteoporosis Foundation (NOF), including all standard elements of a densitometry report such as the t-score, $\mathrm{z}$-score and WHO criteria [31]. From these user entries, the template generates complete sentences automatically. The report can then be easily exported by using a one-click copy-and-paste button (see Figs. 1 and 2).

\section{Creation of radiologic reports}

The FTRs and SRs were created by two radiology residents and two final-year medical students from a single academic medical center in Munich, Germany. They were randomly assigned to two cohorts with each cohort consisting of one resident and one medical student. The residents had 21 and 40 months of experience in radiology each and had previously dictated $25-50$ and less than 25 DXA reports, respectively. The medical students had 2 and 4 months of dedicated experience in radiology and had no prior experience in creating DXA reports. Since our dictation software is personalized and only available for physicians, the medical students had to type their FTRs, whereas the residents dictated theirs.

In an initial training-phase the principle of DXA scans was reviewed and a demonstration of how the SRs can be created using the pre-defined online template was given. Each reader created only one type of report (SR or FTR) for each of the 26 patients. We used a crossover design so that no reader would create more than one report per patient and recall-bias could be avoided. The patients were randomized into two groups of 13 (see Fig. 3a). Then both cohorts began creating reports for the same group of patients. After completing 13 reports, the readers switched to creating the other report type for the remaining 13 patients. They documented the time it took from the moment a DXA scan was opened in the imaging software program until the report was saved.

\section{Evaluation of the reports}

To ensure that potentially reduced reporting times did not come at the cost of lower report quality, we asked two clinicians specializing in internal medicine to rate

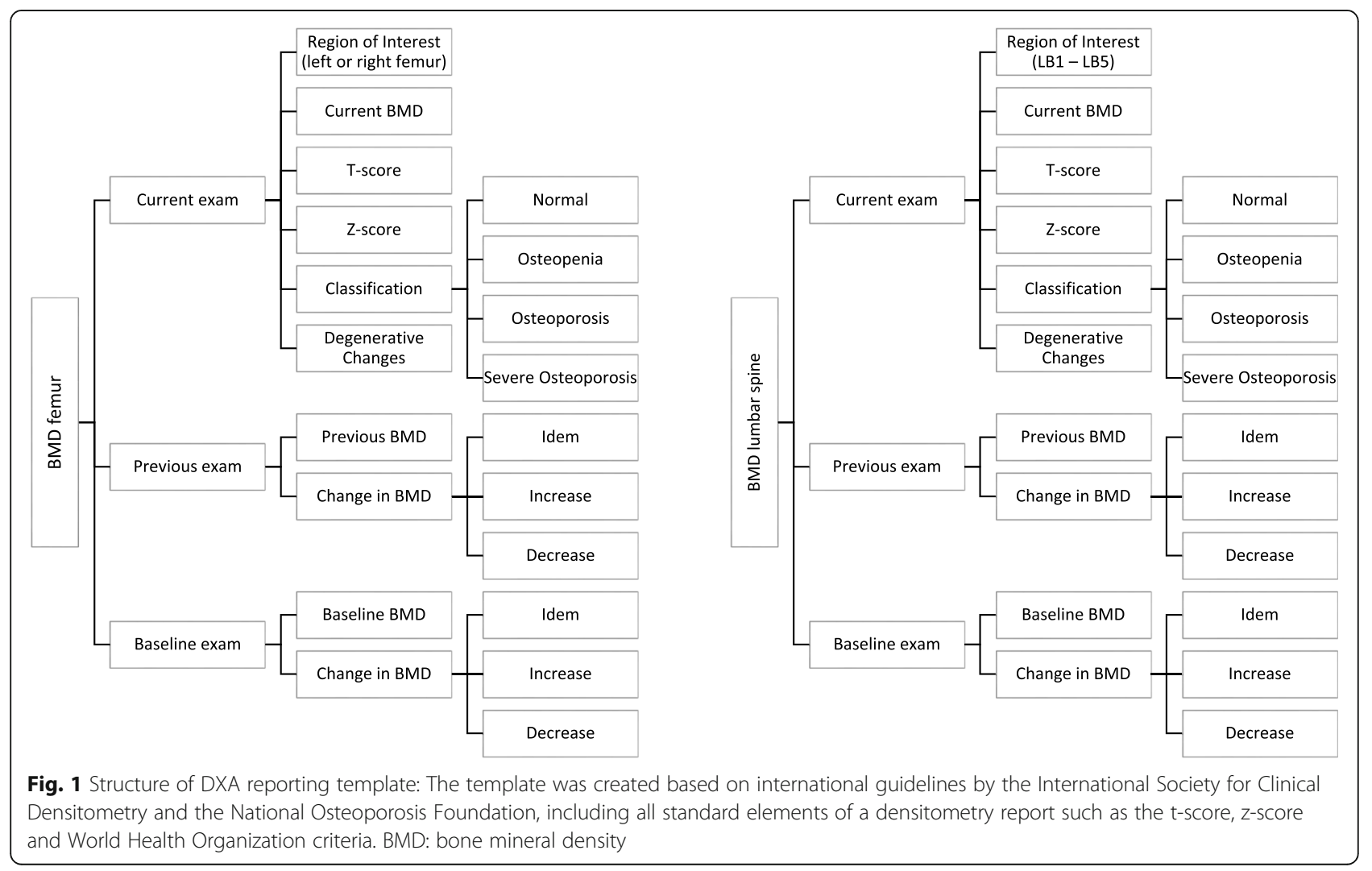




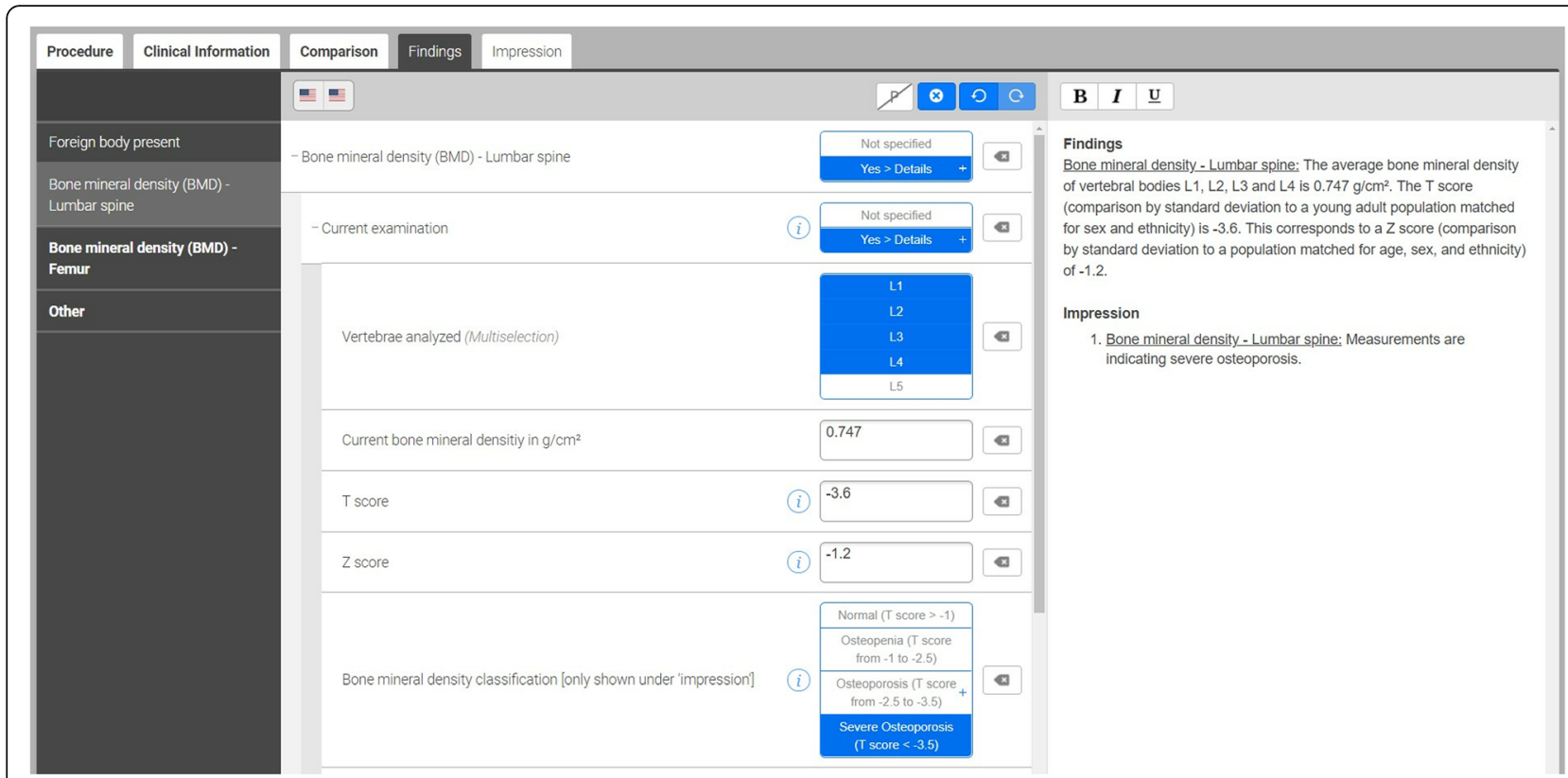

Fig. 2 Screenshot of DXA reporting template: When pre-defined options are selected or values are entered into text fields, the final report text is generated automatically [retrieved from Smart Radiology software, "X-ray bone density" template, on 18 Aug 2018]

the reports (2 and 8 years of experience with patients who require DXA exams).

From each of the two patient groups, six patients were randomly selected, resulting in a total of 12 patients, whose reports were evaluated by the clinicians. There were four reports for each of the 12 patients, adding up to 48 reports in total (see Fig. 3b). These anonymized reports contained the clinical question, a unique report ID, age and gender of the patient. SRs and FTRs were uniformly formatted. The evaluating physicians were blinded to report type and to the level of the reader's experience who had created the report. A standardized questionnaire for DXA report evaluation was provided based on an extensive literature review $[7,10,17,24,34-37]$ to ascertain the most important features of a good radiology report. Among others, content, comprehensibility and clinical relevance were rated on a 10-point Likert scale (see Fig. 4).

\section{Reader's survey}

After completing the reports, the four readers answered an anonymous online survey containing Likert scale and open-ended questions regarding the template and their opinions on structured reporting vs. free-text reporting (see Table in Additional file 3).

\section{Statistical analysis}

Data are reported as medians with interquartile range (IQR) and minimum and maximum for ratings on a 10point Likert scale and as frequencies and percentages for categorical items. The reporting times for each report type were compared separately for residents and medical students (Mann-Whitney-U-test) as voice-recognition software was unavailable to the medical students. To compare reporting times between residents and medical students for the template-based SRs the Wilcoxon signed rank test for paired data was used since one resident and one medical student always created a SR for the same patient. The report ratings by referring physicians were compared using the Wilcoxon signed rank test (Likert scale items and overall rating). The level of significance was defined at $\alpha<0.05$. SPSS Version 20 was used for all statistical calculations.

\section{Results \\ Reporting times}

Both the residents and the medical students required less time for SRs than for FTRs (see Table 1 and Table in Additional file 1). The median reporting time of residents amounted to $4.96 \mathrm{~min}$ for FTRs, compared to 2.71 min for SRs, which corresponds to a reduction of $45.4 \%$ $(P<0.001)$. The effect was even more distinct for the medical students who required $64.4 \%$ less time for SRs than for FTRs (median for FTRs: 7.53 min; median for SRs: $2.68 \mathrm{~min} ; P<0.001)$.

The difference in reporting time between SRs and FTRs was particularly pronounced for patients with more than one previous comparison. In these follow-up exams, residents had a $53.0 \%$ shorter reporting time for SRs than for FTRs (median for FTRs: 6.07 min; median for SRs: $2.85 \mathrm{~min} ; \quad P<0.001$ ) while the reduction 
a
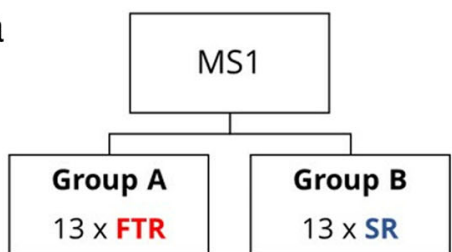

$13 \times$ FTR
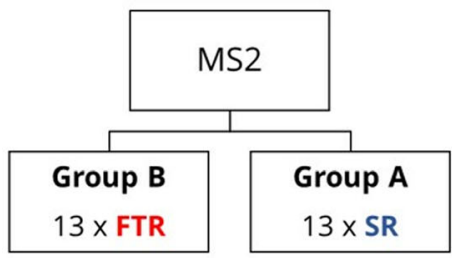

$13 \times \mathrm{SR}$ $=\mathbf{2 6}$ patients

$=26$ reports/examiner

$\begin{array}{ll}2(\mathrm{FTR})+2(\mathrm{SR}) & =4 \text { reports } / \text { patient } \\ 26 \text { patients } \times 4 \text { reports/patient } & =104 \text { reports in total }\end{array}$

$\begin{array}{ll}2(\mathrm{FTR})+2(\mathrm{SR}) & =4 \text { reports } / \text { patient } \\ 26 \text { patients } \times 4 \text { reports/patient } & =104 \text { reports in total }\end{array}$

b

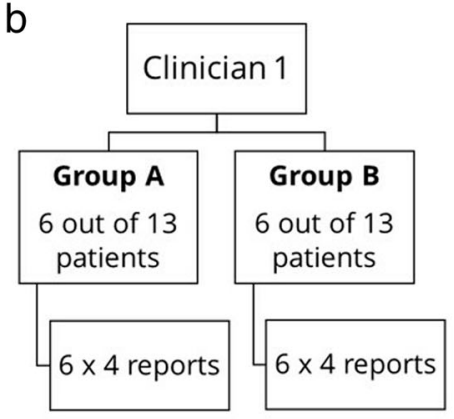

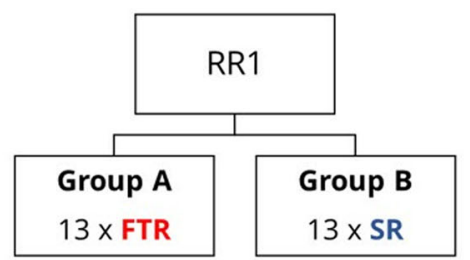

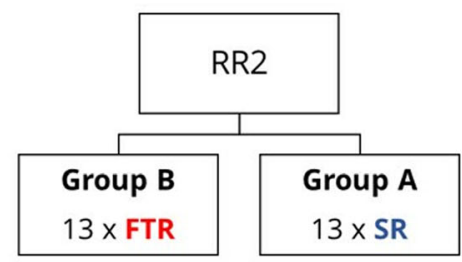

12 patients $\times 4$ reports/patient $=\mathbf{4 8}$ reports in total

2 clinicians $\times 48$ reports/clinician $=96$ evaluations in total

Fig. 3 Study design: A DXA scans of 26 patients were randomized and examined by two medical students and two radiology residents; each reader created either a SR or FTR for each scan B 48 out of 104 reports were evaluated by two clinicians specializing in internal medicine; MS: medical student, RR: radiology resident, FTR: free text report, SR: structured report

for medical students amounted to $66.6 \%$ (median for FTRs: 9.93 min; median for SRs: $3.32 \mathrm{~min} ; P<0.001$ ).

Comparison of reporting times of SRs created by residents vs. medical students showed no significant difference, either for all reports $(P=0.159)$ or for the two subgroups (for single exams: $P=0.969$; for follow-up exams: $P=0.060$ ). Reporting times of FTRs created by residents vs. medical students were not compared since the residents used a free-speech dictation software while the medical students typed their reports.

\section{Report evaluation}

Both FTRs and SRs were evaluated by referring physicians in terms of content, structure, comprehensibility and impact on clinical decision-making (see Table in Additional file 2):

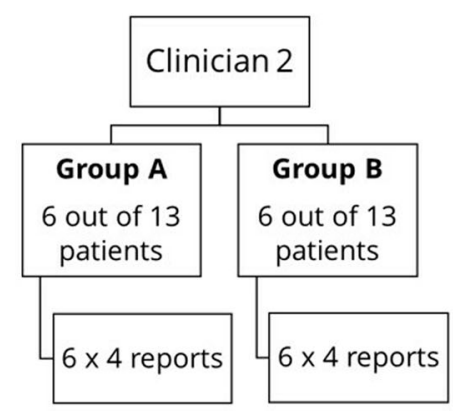

Content and appropriateness of report length

All eight pre-defined key features were included in the content of all FTRs and SRs. With respect to the report extent, all SRs were considered appropriate while only $79.2 \%$ of FTRs received the same rating. Among the remaining FTRs, 14.6\% were rated as "too long" and 6.3\% as "too short". The percentage of FTRs found to have an appropriate extent was equal in both residents and medical students (79.2\% in both). Yet, whereas all remaining FTRs by medical students were viewed as "too long" (20.9\%), the ratings of the remaining FTRs by residents were mixed ("too short": $12.5 \%$, "too long": 8.3\%).

\section{Structure and comprehensibility}

SRs were rated to be significantly superior to FTRs in the extraction of relevant information $(P<0.001)$. Also, 


\section{Content}

The following key items are missing:

$\begin{array}{ll}\text { Femur: } & \\ \text { Side: (left/right) } & \square \\ \text { Current t-value } & \square \\ \text { WHO classification } & \square \\ \text { Bone density over time } & \square \\ & \\ \text { Lumbar spine: } & \square \\ \text { Included vertebrae } & \square \\ \text { Current t-value } & \square \\ \text { WHO classification } & \square \\ \text { Bone density over time } & \end{array}$

Bone density over time

Formal Aspects/ Comprehensibility

( $1=$ completely disagree, $10=$ fully agree $)$

\begin{tabular}{|c|c|c|c|c|c|c|c|c|c|}
\hline Relevant information is extracted quickly. & 1 & 2 & 3 & 4 & 5 & 6 & 7 & 8 & 9 \\
\hline $\begin{array}{l}\text { The structure of the report facilitates information } \\
\text { gain. }\end{array}$ & 1 & 2 & 3 & 4 & 5 & 6 & 7 & 8 & 9 \\
\hline $\begin{array}{l}\text { The language of the report is clear and easy to } \\
\text { understand. }\end{array}$ & 1 & 2 & 3 & 4 & 5 & 6 & 7 & 8 & 9 \\
\hline The extent of the report is: & & & & & & & & $\square$ & \\
\hline
\end{tabular}

Clinical Consequences

(1=completely disagree, $10=$ fully agree $)$

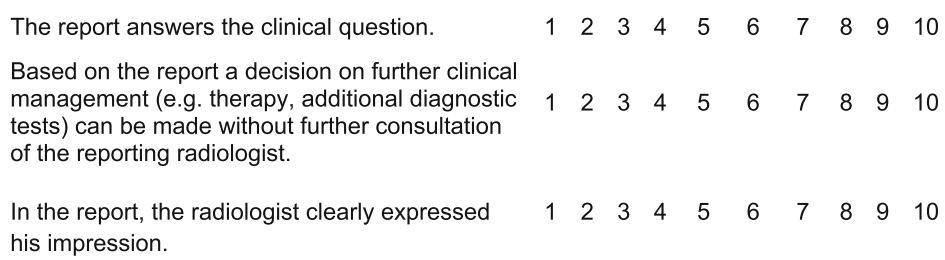

insufficient
poor
medium
high
very high

Fig. 4 Report evaluation questionnaire: This figure shows the report evaluation questionnaire using checkboxes and 10-point Likert scale questions for the three main categories (content, clarity and clinical consequences)

the structure of SRs was found to be more helpful in terms of information gain than that of FTRs $(P<0.001)$, and ratings for comprehensibility were significantly higher for SRs $(P<0.001)$ (see Fig. 5).

\section{Clinical consequences}

SRs were rated to answer the clinical question significantly better than FTRs $(P<0.01)$. SRs and FTRs showed no difference with respect to the contribution to the decision on further clinical management $(P=0.707)$. SRs received significantly higher ratings for the expression of a clear impression $(P<0.01)$.

\section{Overall quality ratings}

Overall quality ratings were significantly higher for SRs vs. FTRs for both residents and medical students. In total, $64.6 \%$ of SRs received ratings of "very high quality" compared to only $16.7 \%$ of FTRs (difference: $+47.9 \%$; $P<0.0001)$. Among the residents, there was an increase of "very high quality" ratings from $25 \%$ for FTRs to $58.3 \%$ for SRs (difference: $+33.3 \%$; $P=0.0010$ ). When comparing ratings by report type created by medical students, only $8.3 \%$ of FTRs were found to be of "very high quality" compared to $70.8 \%$ of SRs (difference: $+62.5 \%$; $P<0.0001$ ) (see Fig. 6). 
Table 1 Reporting times in minutes of radiology residents and final-year medical students for DXA exams

\begin{tabular}{|c|c|c|c|c|c|}
\hline & Median & IQR & Min. & Max. & $P$ \\
\hline \multicolumn{6}{|c|}{ OVERALL AVERAGE $(n=104)$} \\
\hline \multicolumn{6}{|c|}{ Residents $(n=52)$} \\
\hline FTR & 4.96 & $3.98-6.15$ & 2.77 & 7.45 & \\
\hline SR & 2.71 & $2.15-2.90$ & 1.93 & 5.25 & $<0.001$ \\
\hline \multicolumn{6}{|c|}{ Medical students $(n=52)$} \\
\hline FTR & 7.53 & $5.96-10.65$ & 4.45 & 11.90 & \\
\hline SR & 2.68 & $2.33-3.52$ & 1.78 & 7.38 & $<0.001$ \\
\hline \multicolumn{6}{|c|}{ FIRST DXA EXAM $(n=48)$} \\
\hline \multicolumn{6}{|c|}{ Residents $(n=24)$} \\
\hline FTR & 3.83 & $3.14-4.70$ & 2.77 & 5.25 & \\
\hline SR & 2.18 & $2.00-2.75$ & 1.93 & 2.85 & $<0.001$ \\
\hline \multicolumn{6}{|c|}{ Medical students $(n=24)$} \\
\hline FTR & 6.13 & $5.37-6.85$ & 4.45 & 7.63 & \\
\hline$S R$ & 2.33 & $1.99-2.38$ & 1.78 & 4.38 & $<0.001$ \\
\hline \multicolumn{6}{|c|}{ FOLLOW-UP DXA EXAM $(n=56)$} \\
\hline \multicolumn{6}{|c|}{ Residents ( $n=28$ ) } \\
\hline FTR & 6.07 & $5.20-6.70$ & 4.47 & 7.45 & \\
\hline$S R$ & 2.85 & $2.58-3.14$ & 2.17 & 3.14 & $<0.001$ \\
\hline \multicolumn{6}{|c|}{ Medical students $(n=28)$} \\
\hline FTR & 9.93 & $8.55-11.27$ & 5.88 & 11.90 & $<0.001$ \\
\hline SR & 3.32 & $2.92-3.71$ & 2.28 & 7.38 & \\
\hline
\end{tabular}

Median reporting time, interquartile range (IQR), Minimum (Min.), Maximum (Max.), $P$-values for comparisons between free-text reports (FTRs) and structured reports (SRs) using Mann-Whitney U test

\section{Preference of report type}

One resident and both medical students stated structured reporting as their preferred reporting type for DXA examinations. Completeness, shorter reporting times and structure were viewed as the main strengths of SRs. Only the more experienced resident (40 months experience in radiology) preferred free-text reporting to

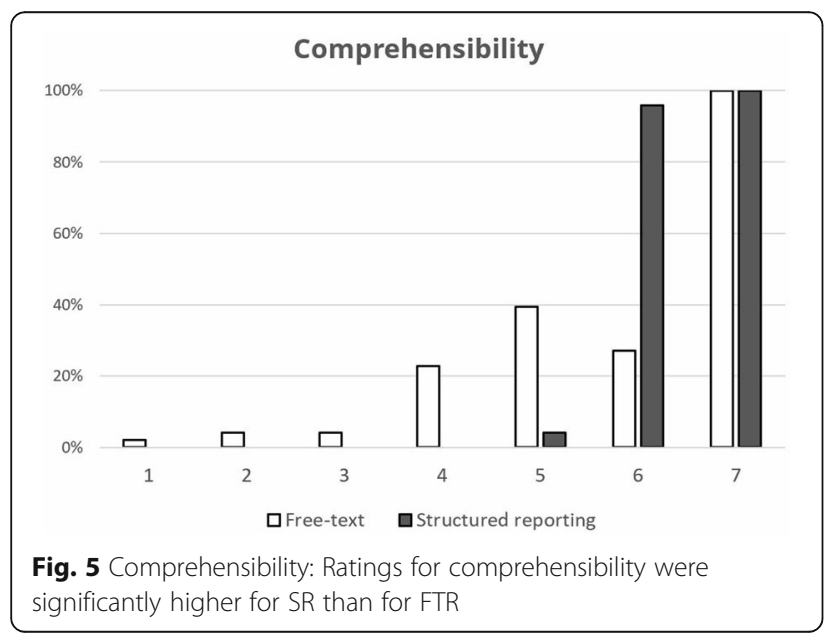

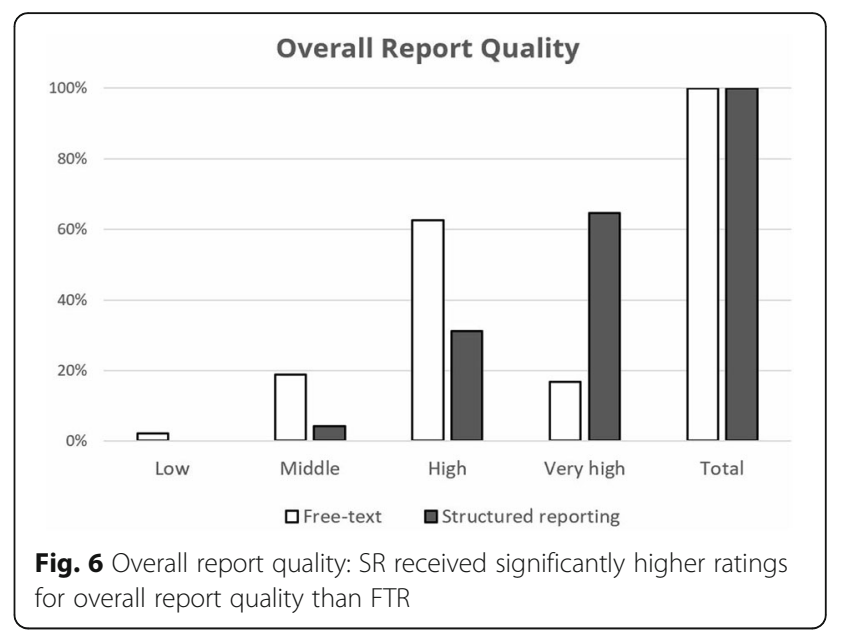

structured reporting, explaining that he enjoyed freespeech dictation more than clicking and entering numbers, and that he did not like to use additional programs. Both evaluating clinicians preferred SRs to FTRs. The structure of SRs was perceived to reduce error rates and facilitate a quick extraction of relevant information.

\section{Discussion}

Template-based structured reports can significantly shorten reporting times and improve report quality

Our findings that SRs exhibit better completeness and result in higher satisfaction of referring physicians are consistent with and extend those from prior reports [6-13, $15,16]$. However, one common concern has been that structured reporting might be more time-consuming and complex than free-text reporting and thus impede productivity $[18,38]$.

Here we provide evidence that SRs may not only lead to better completeness and higher satisfaction of referring physicians but also save time, at least for highly standardized examination types such as DXA. Notably, the more experienced the subject was, the smaller the difference between SRs and FTRs became in terms of reporting time. This observation could be explained by the fact that the medical students typed their FTRs unlike the residents who used the free-speech dictation software. As typing takes more time than free-speech dictation our study might overestimate the difference between the reporting times for medical students and residents. Another explanation could be that residents were more efficient in generating FTRs as they had a more advanced level of experience.

One major advantage of structured reporting is that the user is less prone to make careless mistakes. In this study, for instance, the template not only indicated the reference ranges of the $\mathrm{t}$-score in the decision tree (see Fig. 2), but also performed automatic calculations of the change of bone mineral density over time (in \%). 
Specific DXA report contents that may be missed or incorrectly reported in FTRs include the assessment of BMD changes across non-cross-calibrated machines, fracture risk and vertebral fracture assessment.

All in all, these findings have major medical and economic implications. In the light of the current demographic transition, the workload in radiology is rapidly increasing and structured reporting could make a relevant contribution to improving the efficiency of radiologic workflow. This is particularly true for DXA exams as the prevalence of osteoporosis is increasing in many countries [39-42]. More importantly, structured reporting of DXA exams can make a relevant contribution to improving patient outcome by providing the clinician with more comprehensible reports with higher quality.

\section{Structured reporting as an educational tool for residents and medical students}

The template used in this study reveals that structured reporting can further be a powerful educational tool. Using info boxes within the user interface of the software, relevant background information and exemplary images and reports were displayed. In general, the info boxes might be particularly beneficial to illustrate anatomical images, classifications and up-to-date guidelines. This feature is highly useful for training inexperienced students and residents in a self-guided way. This theory is supported by our finding that the medical students preferred learning DXA reporting with a template and were able to generate very good SRs quickly. However, the medical students also reported that structured reporting might lead to a superficial evaluation due to just clicking through the template, which is a common concern about structured reporting [43]. In the present study, all four readers could easily utilize the software after a short initial training which indicates that only a minimum level of adaptation is required to switch from free-text reporting to structured reporting.

\section{Limitations}

Despite the benefits of structured reporting over freetext reporting highlighted in this study, several limitations need to be acknowledged.

First, due to the retrospective nature of our study, our subjects created the reports in a study setting and not in actual clinical practice. Thus, findings will need to be validated using the template during routine clinical reporting.

Second, in clinical practice there is a broad spectrum of reports (FTR as well as SR) being created. For example, a survey of 265 radiologists in the United States found that only 51\% used structured reporting for at least half of their reports [44]. Another survey in Italy found that $56 \%$ of radiologists never used structured reporting [17]. When it comes to bone density measurements, many centers are still creating FTRs while others have adopted templates like the ISCD's which is essentially a form containing headings and sentences with blanks for the individual BMD, $\mathrm{Z}$ and $\mathrm{T}$-Scores, among others [45]. Furthermore, fully structured online templates with more flexibility, like the one used in this study have been developed. These templates generate sentences automatically in standardized language depending on user entries. Other centers are even attempting to generate their DXA reports fully automatically, although they currently still require revision by radiologists [46]. Given these different approaches to structured reporting of bone density measurements with varying degrees of automation, it might also be beneficial to further evaluate and compare these different types of structured reporting.

Third, the number of subjects who created reports and their experience were limited. A prospective study including a more varied sample of reporting individuals is likely to provide further important insights into the potential of a widespread use of this template. One interesting hypothesis that could be tested is whether the time difference between FTRs and SRs decreases further with increasing experience, although our study indicates that the time required for SRs varies less with experience compared to FTRs.

Additionally, one may argue that the evaluation of report quality is rather subjective and is largely influenced by the evaluating clinician, potentially limiting the generalizability of our findings. Evaluations of the report quality by a larger, more diverse group of clinicians may be beneficial. However, due to the highly standardized nature of DXA exams, we believe that the quality ratings are likely to be consistent even among many referring physicians.

Finally, the observations of the present study cannot be generalized to other radiology examinations and their reports. Reporting times might be less likely to be improved by structured reporting in less standardized, highly variable exams, since a much more complex template structure would be required. But at the same time, reports of highly complex exams might especially benefit from the guidance of a template, since SRs were shown to exhibit higher completeness and allow better extraction of information [6-13]. Importantly, the extent to which structured reporting can improve reporting efficiency and quality strongly depends on the technical features of the utilized software. For instance, an automatic insertion of technical details into the radiology report was found to significantly improve report accuracy [47]. In a similar manner, features such as an automatic insertion of references to previous reports or an automatic identification of certain types of information could create added value, even for less standardized exam. 
Further evaluation of different types of structured reporting templates in prospective (multicenter) studies with readers at various levels of experience and a larger number of evaluating physicians is likely to provide a broader impression.

\section{Conclusion}

In highly standardized exams such as radiographic bone density measurements, template-based structured reporting might lead to shorter reporting times. At the same time, structured reporting may improve report quality and serve as an effective educational tool for medical students and radiology residents during their training.

\section{Supplementary information}

Supplementary information accompanies this paper at https://doi.org/10. 1186/s12891-020-03200-w.

Additional file 1. Reporting times for DXA exams.

Additional file 2. Report evaluation by referring clinicians.

Additional file 3. Reader's survey.

\section{Abbreviations}

DXA: Dual X-ray absorptiometry; FTR: Free-text report; SR: Structured report: ISCD: The International Society for Clinical Densitometry

\section{Acknowledgements}

None.

\section{Authors' contributions}

SK: primary author, data analysis and interpretation. LS: primary author, data collection, analysis and interpretation. JE, AC, FC, EK, MG, RB, FM: participated in study, revision of scientific content. WS, NS, FG: conceived ideas / study design, revisions of scientific content. The author(s) read and approved the final manuscript.

\section{Funding}

The authors received no specific funding for this work.

\section{Availability of data and materials}

The datasets used and/or analysed during the current study are available from the corresponding author on reasonable request.

\section{Ethics approval and consent to participate}

Not applicable.

\section{Consent for publication}

Not applicable.

\section{Competing interests}

The following authors of this manuscript declare relationships with Smart Reporting $\mathrm{GmbH}$ (online software company for structured reporting templates):

- Su Hwan Kim: created and reviewed structured templates.

- Lara Sobez: created structured templates.

- Franziska Galiè: created and reviewed structured templates, background research.

- Nora Sommer: created and reviewed structured templates, background research.

- Wieland Sommer: co-founder.

None of the other authors declare any conflict of interest relevant to the content of this study.

\section{Author details}

${ }^{1}$ Department of Radiology, University Hospital, LMU Munich, Munich, Germany. ${ }^{2}$ Munich Transplant Center, University Hospital, LMU Munich, Munich, Germany. ${ }^{3}$ Department of Internal Medicine III, University Hospital, LMU Munich, Munich, Germany. ${ }^{4}$ Department of Radiation Oncology, University Hospital, LMU Munich, Munich, Germany. ${ }^{5}$ Department of Diagnostic and Interventional Radiology, Paediatric Radiology and Neuroradiology, University Medical Center Rostock, Rostock, Germany.

Received: 3 February 2020 Accepted: 10 March 2020

Published online: 16 April 2020

\section{References}

1. Gunderman RB, McNeive LR. Is structured reporting the answer? Radiology. 2014:273(1):7-9.

2. Radiology ESo. Good practice for radiological reporting. Guidelines from the European Society of Radiology (ESR). Insights Imaging. 2011;2(2):93-6.

3. Morgan TA, Helibrun ME, Kahn CE. Reporting initiative of the Radiological Society of North America: progress and new directions. Radiology. 2014; 273(3):642-5.

4. Dunnick NR, Langlotz CP. The radiology report of the future: a summary of the 2007 intersociety conference. J Am Coll Radiol. 2008;5(5):626-9.

5. KSAR SGFRC. Essential items for structured reporting of rectal Cancer MRI: 2016 consensus recommendation from the Korean Society of Abdominal Radiology. Korean J Radiol. 2017;18(1):132-51.

6. Norenberg D, Sommer WH, Thasler W, D'Haese J, Rentsch M, Kolben T, Schreyer A, Rist C, Reiser M, Armbruster M. Structured Reporting of RectalMagnetic Resonance Imaging in Suspected Primary Rectal Cancer: Potential Benefits for Surgical Planning and Interdisciplinary Communication. Investigative radiology. 2017:52(4):232-39.

7. Gassenmaier S, Armbruster M, Haasters F, Helfen T, Henzler T, Alibek S, Pforringer D, Sommer WH, Sommer NN. Structured reporting of MRI of the shoulder - improvement of report quality? Eur Radiol. 2017;27(10):4110-9.

8. Brook OR, Brook A, Vollmer CM, Kent TS, Sanchez N, Pedrosa I. Structured reporting of multiphasic $C T$ for pancreatic cancer: potential effect on staging and surgical planning. Radiology. 2015;274(2):464-72.

9. Sahni VA, Silveira PC, Sainani NI, Khorasani R. Impact of a structured report template on the quality of MRI reports for rectal Cancer staging. AJR Am J Roentgenol. 2015;205(3):584-8.

10. Schwartz LH, Panicek DM, Berk AR, Li Y, Hricak H. Improving communication of diagnostic radiology findings through structured reporting. Radiology. 2011;260(1):174-81.

11. Sabel BO, Plum JL, Kneidinger N, Leuschner G, Koletzko L, Raziorrouh B, Schinner R, Kunz WG, Schoeppe F, Thierfelder KM, et al. Structured reporting of CT examinations in acute pulmonary embolism. J Cardiovasc Comput Tomogr. 2017:11(3):188-95.

12. Wildman-Tobriner B, Allen BC, Bashir MR, Camp M, Miller C, Fiorillo LE, Cubre A, Javadi S, Bibbey AD, Ehieli WL, et al. Structured reporting of $C T$ enterography for inflammatory bowel disease: effect on key feature reporting, accuracy across training levels, and subjective assessment of disease by referring physicians. Abdom Radiol (NY). 2017;42(9):2243-50.

13. Flusberg M, Ganeles J, Ekinci T, Goldberg-Stein S, Paroder V, Kobi M, Chernyak $V$. Impact of a structured report template on the quality of $C T$ and MRI reports for hepatocellular carcinoma diagnosis. J Am Coll Radiol. 2017; 14(9):1206-11.

14. Dickerson E, Davenport MS, Syed F, Stuve O, Cohen JA, Rinker JR, Goldman MD, Segal BM, Foerster BR, Michigan Radiology Quality C. Effect of template reporting of brain MRIs for multiple sclerosis on report thoroughness and neurologist-rated quality: results of a prospective quality improvement project. J Am Coll Radiol. 2017;14(3):371-9 e371.

15. Schoeppe F, Sommer WH, Haack M, Havel M, Rheinwald M, Wechtenbruch J, Fischer MR, Meinel FG, Sabel BO, Sommer NN. Structured reports of videofluoroscopic swallowing studies have the potential to improve overall report quality compared to free text reports. Eur Radiol. 2018:28(1):308-15.

16. Schoeppe F, Sommer WH, Norenberg D, Verbeek M, Bogner C, Westphalen $C B$, Dreyling M, Rummeny EJ, Fingerle AA. Structured reporting adds clinical value in primary $C T$ staging of diffuse large B-cell lymphoma. Eur Radiol. 2018;28(9):3702-9.

17. Faggioni L, Coppola F, Ferrari R, Neri E, Regge D. Usage of structured reporting in radiological practice: results from an Italian online survey. Eur Radiol. 2017;27(5):1934-43. 
18. Weiss $D L$, Langlotz CP. Structured reporting: patient care enhancement or productivity nightmare? Radiology. 2008;249(3):739-47.

19. Hangiandreou NJ, Stekel SF, Tradup DJ. Comprehensive clinical implementation of DICOM structured reporting across a radiology ultrasound practice: lessons learned. J Am Coll Radiol. 2017;14(2):298-300.

20. Reiner Bl. Optimizing technology development and adoption in medical imaging using the principles of innovation diffusion, part l: theoretical, historical, and contemporary considerations. J Digit Imaging. 2011;24(5): 750-3.

21. Ganeshan D, Duong PT, Probyn L, Lenchik L, McArthur TA, Retrouvey M, Ghobadi EH, Desouches SL, Pastel D, Francis IR. Structured reporting in radiology. Acad Radiol. 2018;25(1):66-73.

22. Towbin AJ, Hawkins CM. Use of a web-based calculator and a structured report generator to improve efficiency, accuracy, and consistency of radiology reporting. J Digit Imaging. 2017;30(5):584-8.

23. Hanna TN, Shekhani H, Maddu K, Zhang C, Chen Z, Johnson JO. Structured report compliance: effect on audio dictation time, report length, and total radiologist study time. Emerg Radiol. 2016;23(5):449-53.

24. Segrelles JD, Medina R, Blanquer I, Marti-Bonmati L. Increasing the efficiency on producing radiology reports for breast Cancer diagnosis by means of structured reports. A comparative study. Methods Inf Med. 2017;56(3):248-60.

25. Li N, Li XM, Xu L, Sun WJ, Cheng XG, Tian W. Comparison of QCT and DXA: osteoporosis detection rates in postmenopausal women. Int J Endocrinol. 2013;2013:895474

26. Khoo BC, Brown K, Cann C, Zhu K, Henzell S, Low V, Gustafsson S, Price Rl, Prince RL. Comparison of QCT-derived and DXA-derived areal bone mineral density and T scores. Osteoporos Int. 2009;20(9):1539-45.

27. Lukaszewicz A, Uricchio J, Gerasymchuk G. The art of the radiology report: practical and stylistic guidelines for perfecting the conveyance of imaging findings. Can Assoc Radiol J. 2016;67(4):318-21.

28. Morgan SL, Prater GL. Quality in dual-energy X-ray absorptiometry scans. Bone. 2017;104:13-28.

29. Punda M, Grazio S. Bone densitometry--the gold standard for diagnosis of osteoporosis. Reumatizam. 2014;61(2):70-4.

30. Dimai HP. Use of dual-energy X-ray absorptiometry (DXA) for diagnosis and fracture risk assessment; WHO-criteria, T- and Z-score, and reference databases. Bone. 2017;104:39-43.

31. Lewiecki EM, Binkley N, Morgan SL, Shuhart CR, Camargos BM, Carey JJ, Gordon CM, Jankowski LG, Lee JK, Leslie WD, et al. Best practices for dualenergy X-ray absorptiometry measurement and reporting: International Society for Clinical Densitometry Guidance. J Clin Densitom. 2016;19(2):127-40.

32. Krueger D, Shives E, Siglinsky E, Libber J, Buehring B, Hansen KE, Binkley N. DXA Errors Are Common and Reduced by Use of a Reporting Template. Journal of Clinical Densitometry. 2019;22(1):115-24.

33. 2019 ISCD Official Positions - Adult [https://www.iscd.org/official-positions/2 019-iscd-official-positions-adult/]. Accessed 6 Mar 2020

34. Wallis A, McCoubrie $P$. The radiology report--are we getting the message across? Clin Radiol. 2011;66(11):1015-22

35. Rothman M. Malpractice issues in radiology: radiology reports. AJR Am J Roentgenol. 1998;170(4):1108-9.

36. Yang C, Kasales CJ, Ouyang T, Peterson CM, Sarwani NI, Tappouni R, Bruno M. A succinct rating scale for radiology report quality. SAGE Open Med. 2014;2:2050312114563101.

37. Wallis A, Edey A, Prothero D, McCoubrie P. The Bristol radiology report assessment tool (BRRAT): developing a workplace-based assessment tool for radiology reporting skills. Clin Radiol. 2013;68(11):1146-54.

38. Bosmans JM, Peremans L, Menni M, De Schepper AM, Duyck PO, Parizel PM Structured reporting: if, why, when, how-and at what expense? Results of a focus group meeting of radiology professionals from eight countries. Insights Imaging. 2012;3(3):295-302.

39. Wright NC, Looker AC, Saag KG, Curtis JR, Delzell ES, Randall S, DawsonHughes B. The recent prevalence of osteoporosis and low bone mass in the United States based on bone mineral density at the femoral neck or lumbar spine. J Bone Miner Res. 2014;29(11):2520-6.

40. Lotters FJ, van den Bergh JP, de Vries F, Rutten-van Molken MP. Current and future incidence and costs of osteoporosis-related fractures in the Netherlands: combining claims data with BMD measurements. Calcif Tissue Int. 2016;98(3):235-43.

41. Khadilkar AV, Mandlik RM. Epidemiology and treatment of osteoporosis in women: an Indian perspective. Int J Women's Health. 2015;7:841-50.
42. Chen P, Li Z, Hu Y. Prevalence of osteoporosis in China: a meta-analysis and systematic review. BMC Public Health. 2016;16(1):1039.

43. Srinivasa Babu A, Brooks ML. The malpractice liability of radiology reports: minimizing the risk. Radiographics. 2015;35(2):547-54.

44. Powell DK, Silberzweig JE. State of structured reporting in radiology, a survey. Acad Radiol. 2015;22(2):226-33.

45. US Adult DXA Sample Report [https://iscd.app.box.com/v/US-Adult-DXASample-Report]. Accessed 30 Oct 2018.

46. Tsai IT, Tsai MY, Wu MT, Chen CK. Development of an automated bone mineral density software application: facilitation radiologic reporting and improvement of accuracy. J Digit Imaging. 2016;29(3):380-7.

47. Abujudeh HH, Govindan S, Narin O, Johnson JO, Thrall JH, Rosenthal DI. Automatically inserted technical details improve radiology report accuracy. Am Coll Radiol. 2011;8(9):635-7.

\section{Publisher's Note}

Springer Nature remains neutral with regard to jurisdictional claims in published maps and institutional affiliations.
Ready to submit your research? Choose BMC and benefit from:

- fast, convenient online submission

- thorough peer review by experienced researchers in your field

- rapid publication on acceptance

- support for research data, including large and complex data types

- gold Open Access which fosters wider collaboration and increased citations

- maximum visibility for your research: over $100 \mathrm{M}$ website views per year

At BMC, research is always in progress.

Learn more biomedcentral.com/submissions 